stock recovery unless the International Commission for the Conservation of Atlantic Tunas (ICCAT), which sets the quotas, reforms the ineffective 15 -year recovery plan it adopted in 2006.

When it meets in November this year, the ICCAT must adopt the measures advocated by its own Standing Committee on Research and Statistics (http://iccat.int/scrs.htm). These include closure of the Mediterranean throughout the spawning season, full enforcement of the increased minimum size for caught tuna, and a catch limit of 15,000 tonnes - less than a third of recent totals - for the next few years. It should also heed the call of the conservation group WWF, which is asking for a three-year moratorium on bluefin tuna fishing (http://tinyurl.com/5ehxtw). With far too many fishing vessels, significant underreporting of catches by several EU countries and considerable illegal, unregulated and unreported fishing by neighbouring states such as Turkey, Croatia and Libya, time is fast running out for the Mediterranean bluefin tuna. Its loss would be an economic and ecological disaster. Geoff Arnold is former Chair of the Fisheries Science and Management Group at the Centre for Environment, Fisheries and Aquaculture Science (CEFAS), Lowestoft, UK, and a member of the SCOR Panel on New Technologies for Observing Marine Life.

e-mail: geoff@arnold45.freeserve.co.uk

\title{
Ocean exhibit makes waves
}

The Sant Ocean Hall

Smithsonian National Museum of Natural History, Washington DC

Opens on 27 September 2008

Geographers will tell you that there are four world oceans, or five if you count Antarctic waters. The designers of the new Sant Ocean Hall at the Smithsonian National Museum of Natural History, based in Washington DC, beg to differ. Although it acknowledges distinct ocean basins, the exhibit emphasizes that the world's waters are linked in a single ecosystem that influences all life on Earth.

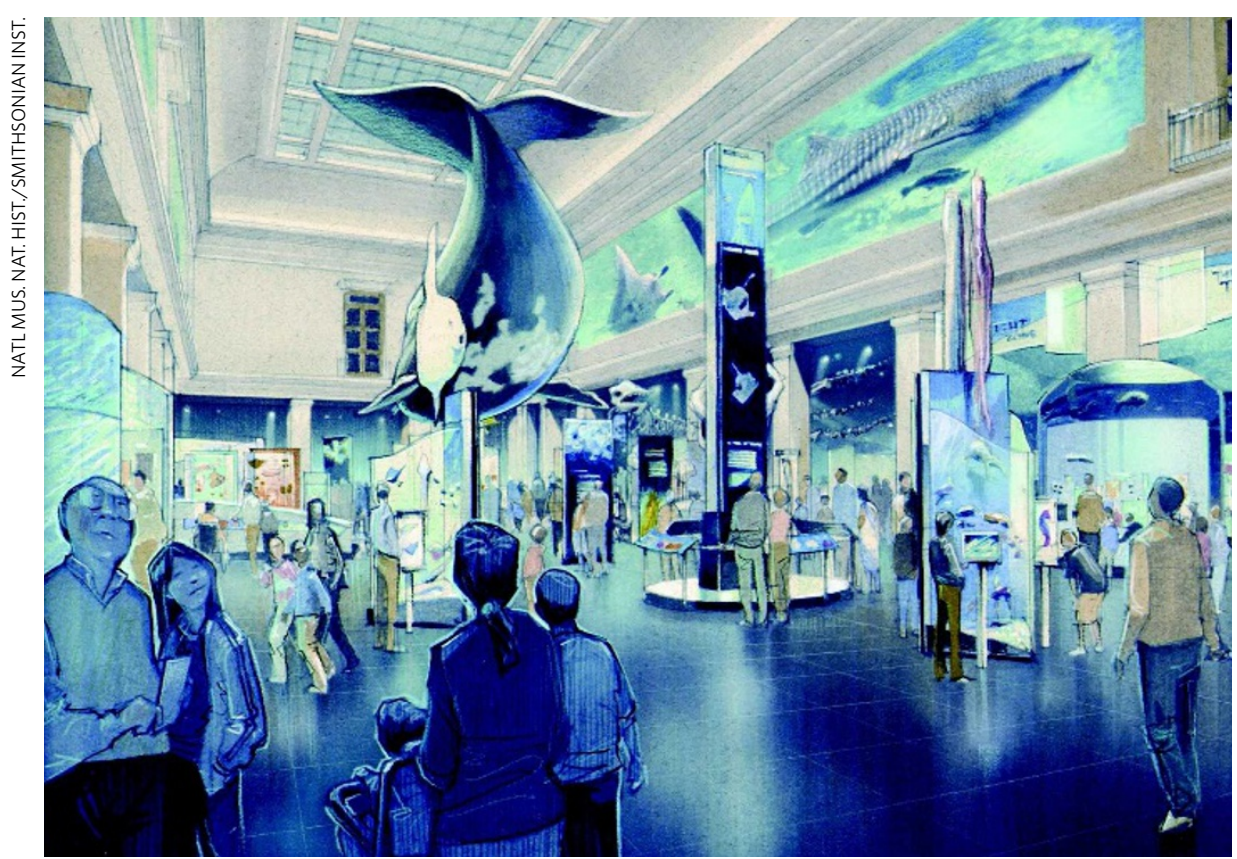

An artist's impression of the Sant Ocean Hall shows its emphasis on the oceans as a single ecosystem.
2001 discovery of a strange squid with spaghetti-like arms. A news kiosk features frequent ocean updates. Exhibit developer Jill Johnson hopes the displays will educate visitors and encourage them to be better stewards of the world's waters.

Looming over the hall is a full-scale model of a female North Atlantic right whale called Phoenix. Scientists have spent around 20 years tracking the real Phoenix, distinguishable by her unique scars. The 14-metre-long mother of three was recently spotted in the Gulf of Maine, and is one of an estimated 350 North Atlantic right whales left in the ocean.

One of the strengths of the Smithsonian is its vast collection of marine species, which the hall's curators were eager to display. Specimens are traditionally stored in alcohol, but a change in the fire regulations in 2005 meant that firesafety experts would allow only a maximum of 38 litres of alcohol in the 2,000-metre-square space, explains Johnson.

The answer came from technology company $3 \mathrm{M}$, based in St Paul, Minnesota. One of their non-flammable, non-toxic hydrofluoroether fluids, developed as a cleaning fluid and solvent, forms an envelope around samples to seal in the formalin preservative. $3 \mathrm{M}$ donated some 4,000 litres of the stuff to the museum, where it now surrounds various artefacts, including a coelacanth and her pup, a prehistoric fish that was thought to be extinct until its rediscovery in 1938.

The hall's specimens are the first to be displayed in the fluid, which, unlike alcohol, does not leach colour. "A lot of other museums are really interested in how this works out," Vecchione says.

One of the hall's stars is a seven-metrelong giant squid that rests majestically in a coffin-like tank filled with the liquid. Along with a three-metre-long male squid, it is on loan from the Spanish research organization CEPESMA. Transporting the pair across the Atlantic in 1,500 litres of formalin was quite a task. The US Air Force and Navy helped out with the trip, dubbed 'Operation Calamari'. Elizabeth Musteen, who coordinated the journey, was concerned about the weight of the cargo until an unimpressed Air Force representative chastised her: "Ma'am, I move tanks. I can do this."

Also on show is a hand-carved Tlingit canoe, some 8 metres long. The Pacific northwest tribe is one of many cultures represented in the hall that depend on the sea. As Johnson explains, "The main message is that the ocean, singular, is a global system that is essential to all life — including yours".

Amber Dance is a News intern based in Nature's Washington DC office. 